\title{
Imaging Nanoscale Optical Fields with Inelastic Electron-light Scattering
}

Tyler Harvey ${ }^{1}$, Jan-Wilke Henke ${ }^{2}$, Ofer Kfir ${ }^{2}$, Murat Sivis ${ }^{2}$, Hugo Lourenço-Martins ${ }^{2}$, Armin Feist ${ }^{2}$ and Claus Ropers ${ }^{2}$

${ }^{1}$ National Center for Electron Microscopy, Molecular Foundry, Lawrence Berkeley National Laboratory, Berkeley, California, United States, ${ }^{2}$ University of Göttingen, Göttingen, Niedersachsen, Germany

Although absorption or emission of a single photon by an electron is forbidden in free space by energymomentum conservation, this interaction is possible in the presence of a material. The breaking of translation symmetry allows for coupling between electron momentum and the electromagnetic field amplitude [1]. The strength of this coupling, $\beta$, depends on the shape and optical response of the material as well as the incident optical power [2]. Because electron beams can be focused to sub-nanometer spots in modern scanning transmission electron microscopes, it is also possible to map inelastic electron-light scattering on the same length scale (see Fig. 1). This technique, called photon-induced near-field electron microscopy (PINEM) [1], can be employed to image optical modes and other nanoscale optical fields with nanometer spatial resolution.

The signal-to-noise ratio of PINEM can be very high as compared to EELS for low-loss excitations, as a nearly empty zero-loss peak and high occupation of peaks corresponding to one or more inelastic scattering events occurs with easily reachable incident optical power on many specimens. PINEM has been employed to image proteins [3], cells [3,4], single-atom-high step-edges [5], confined [6] and propagating [7] surface plasmons, and nanoparticles [8]. As PINEM has been typically measured in an ultrafast transmission electron microscopes with femtosecond temporal resolution [9], it is also straightforward to map the temporal response of optically excited states.

We apply this technique to study three materials: chiral nanostructures (Fig. 2), nanostars with high field enhancement, and microspheres which support whispering gallery modes [10]. By varying the timing, polarization and the wavelength of the illuminating light, we gain access to information about symmetries, temporal behavior of long-lifetime optical resonances, and other details that are challenging or impossible to probe in electron energy loss spectroscopy without a driving optical field [11]. 


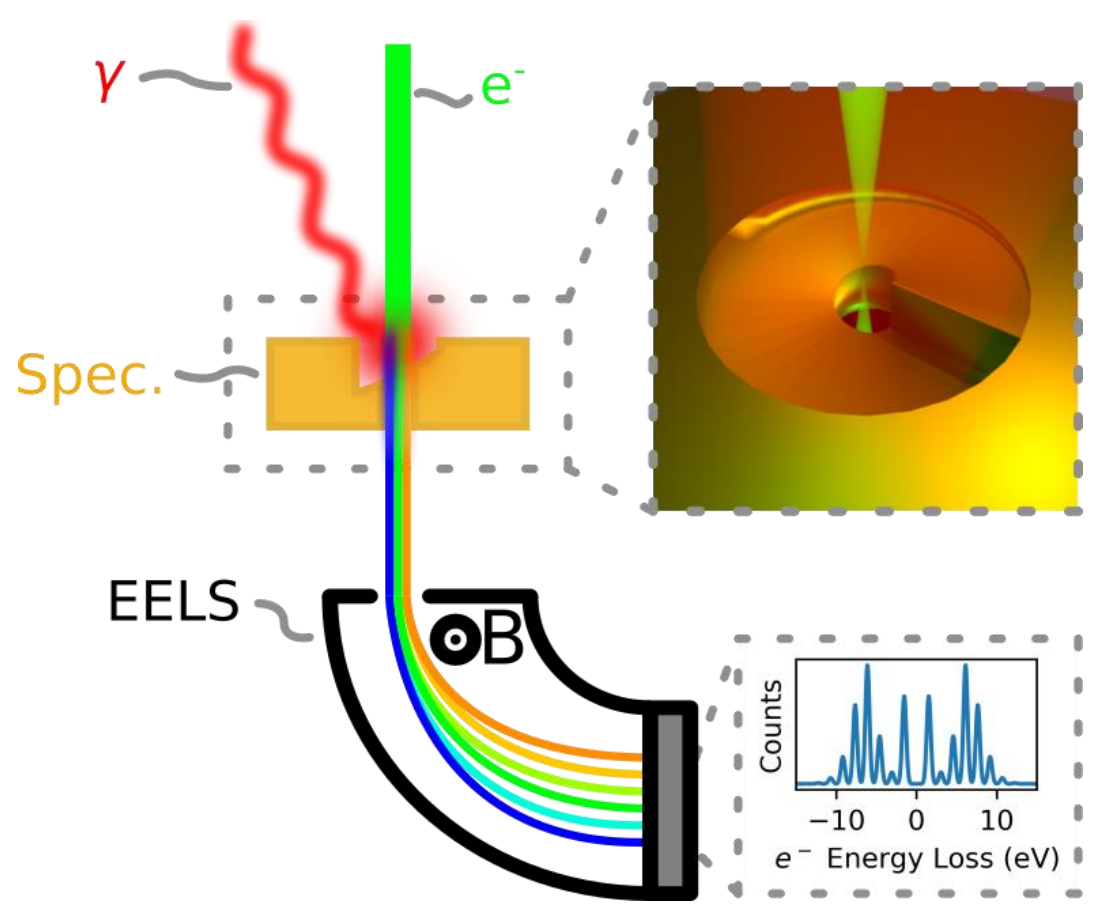

Figure 1. Illustration depicting a PINEM experiment. An optical beam ( $\gamma$, red) illuminates specimen (spec., gold), while the electron beam (e-, green) exchanges quanta of momentum and energy with the induced field around the specimen.

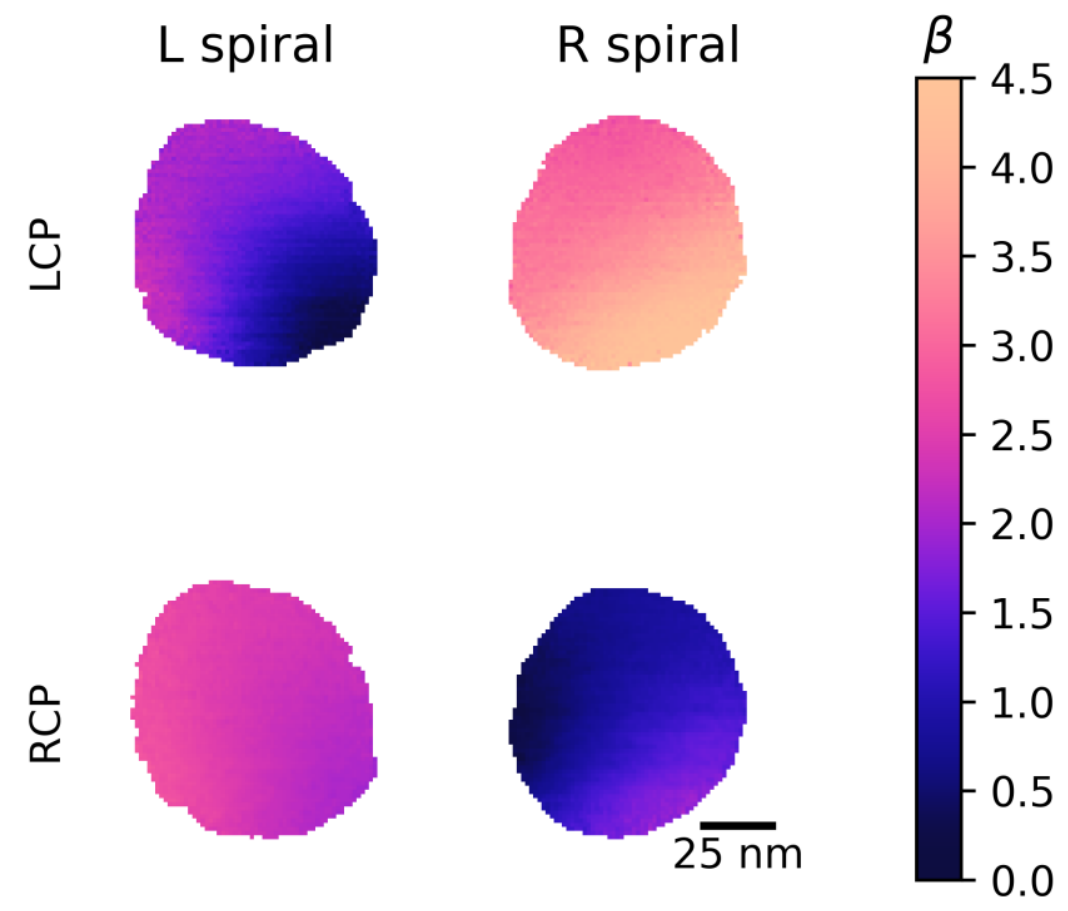

Figure 2. Measured maps of the coupling constant $\beta$ across holes in the center of left-handed (L spiral) and right-handed ( $\mathrm{R}$ spiral) chiral nanostructures excited by left-circularly-polarized (LCP) or rightcircularly-polarized (RCP) light. The optical response across the hole is much stronger for one circular polarization, and the relationship flips when we flip the handedness of the structure, demonstrating that we can obtain 3D structural information from a 2D image. 


\section{References}

[1] B. Barwick et al., Nature 462 (2009) p. 902.

[2] A. Feist et al., Nature 521 (2015) p. 200.

[3] D. J. Flannigan et al., PNAS 107, (2010) p. 9933.

[4] M. Kaplan et al., Ang. Chem. Intl. Ed. 56 (2017) p. 11498.

[5] S.T. Park et al., PNAS 110 (2013) p. 9277.

[6] L. Piazza et al., Nat. Comm. 6 (2015) p. 6407.

[7] I. Madan et al., Sci. Adv. 5 (2019) p. eaav8358.

[8] A. Yurtsever et al., Nano Lett. 12 (2012) p. 3334.

[9] A. Feist et al., Ultramicroscopy 176 (2017) p. 63.

[10] O. Kfir et al., arXiv:1910.09540.

[11] T.R.H acknowledges the support of a postdoctoral fellowship from Alexander von Humboldt Foundation and its sponsor, the German Federal Ministry for Education and Research. This work was also supported by the Deutsche Forschungsgemeinschaft (DFG-SFB 1073/project A05). 